\title{
Anti-Tubercular Treatment of Tuberculous Meningitis Cause Paradoxical Multiple Neurotuberculoma
}

\author{
Kumar $\mathrm{MK}^{1}$, Kumar $\mathrm{P}^{2}$, Singh $\mathrm{A}^{3}$
}

\begin{abstract}
Tuberculosis is the most common cause of infectious disease related mortality worldwide. Paradoxical reactions to antituberculous treatment have created difficulties in the management of extrapulmonary tuberculosis, particularly central nervous system tuberculosis. We report a three years baby with tuberculous meningitis, who was treated outside with anti-tuberculous drugs without steroid one month back and presented in our emergency as status epilepticus. This case emphasizes the importance of addition of steroid along with anti-tubercular drugs in the treatment of central nervous system tuberculosis.
\end{abstract}

Key words: Anti- tuberculous treatment, Neurotuberculoma, Paradoxical response, Tuberculous Meningitis.

\section{Introduction}

ndia had an estimated 2.3 million (26\% of global burden) Tuberculosis (TB) cases in 2010, and ranked $16^{\text {th }}$ in terms of incidence rate amongst 22 highest TB burden countries ${ }^{1]}$. Tuberculosis is the most common cause of infectious disease related mortality worldwide. Paradoxical reactions to anti-tuberculous therapy (ATT) have created difficulties in the management of extra pulmonary tuberculosis, particularly central nervous system tuberculosis (CNS TB). We report a 3 years old baby with tuberculous meningitis, who was treated outside with anti-tuberculous drugs without steroid one month back and presented in our emergency as status epilepticus.

\section{The Case}

Three years old baby presented to emergency department with repeated convulsive movement of the whole body for past six hours. Each convulsive episode persisted for 10 minutes, which was associated with uprolling of the eye ball and loss of consciousness. There was history of vomiting one episode day before onset of seizure. There was no history of fever. Four weeks ago the patient was diagnosed as tuberculous meningitis in a private hospital on the basis of prolonged fever, vomiting, convulsion, positive tuberculin test, history of tuberculous contact, positive meningeal signs and cerebrospinal fluid analysis. Her initial computed tomography (CT) Scan brain did not showed any ring enhancing lesion (Figure 1).
'Dr. Mani Kant Kumar, MBBS, MD, Associate Professor, ${ }^{2}$ Dr. Prashant Kumar, MBBS, MD, Assistant Professor, ${ }^{3}$ Dr. Anjali Singh, MBBS, Senior Resident. All from the Department of Paediatrics, Narayan Medical College and Hospital, Jamuhar, Sasaram, Bihar, India.

\section{Address for correspondence: \\ Dr. Mani Kant Kumar \\ E-mail: manikant7@yahoo.com}

\section{How to cite}

Kumar MK, Kumar P, Singh A. Anti-Tubercular Treatment of Tuberculous Meningitis Cause Paradoxical Multiple Neurotuberculoma. J Nepal Paediatr Soc 2014;34(3):236-238.

doi: http://dx.doi.org/10.3126/jnps.v34i3.10752

This work is licensed under a Creative Commons Attribution 3.0 License.

\section{(c) (i)}

She was started on 4 drugs ATT without steroid consisting of isoniazid, rifampicin, pyrazinamide and ethambutol as intensive phase for two months, all taken once daily in morning empty stomach per orally. She was also given phenytoin@5mg/kg/day per orally. During first four weeks of treatment, there was improvement in symptoms. On examination she was tachycardic with heart rate of 102/ minute, and altered sensorium with Glasgow coma scale (GCS) of 12 . Other vital parameter were within normal limits. On central nervous system examination there were brisk deep tendon reflexes, Bibinski positive bilaterally and power and tone were normal. Complete blood count, routine biochemistry, serum electrolyte level were normal. Repeat CT Scan Brain 
revealed multiple ring enhancing lesion (Inflammatory granuloma) as shown in Figure 2.

In our Institute patient was managed with intra venous (IV) anti-epileptics Diazepam, followed by loading dose of Intra venous phenytoin as per protocol, seizure controlled and later changed to oral maintenance dose of phenytoin once daily, steroid was added (Initially IV Dexamethasone for seven days then shifted to oral prednisone@ 2mg/ kg/day in three divided dose for six weeks and tappered off over two weeks) and ATT were continued as per earlier regimen. Patient gradually improved without any recurrence of seizure.

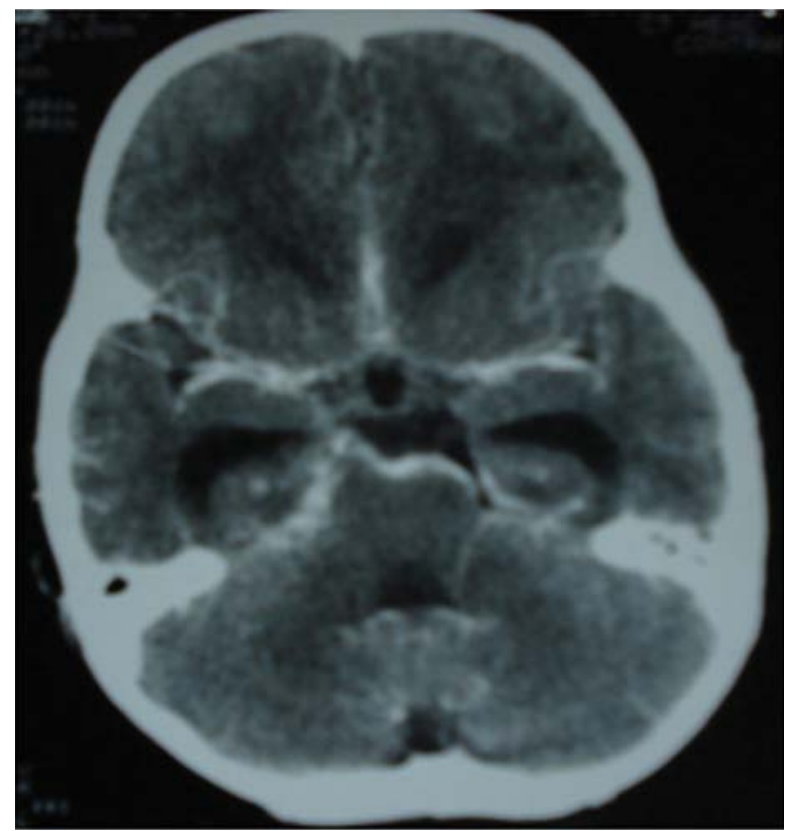

Fig 1: CECT Brain at the time of diagnosis of TBM Showed basal exudates and mild ventriculomegaly

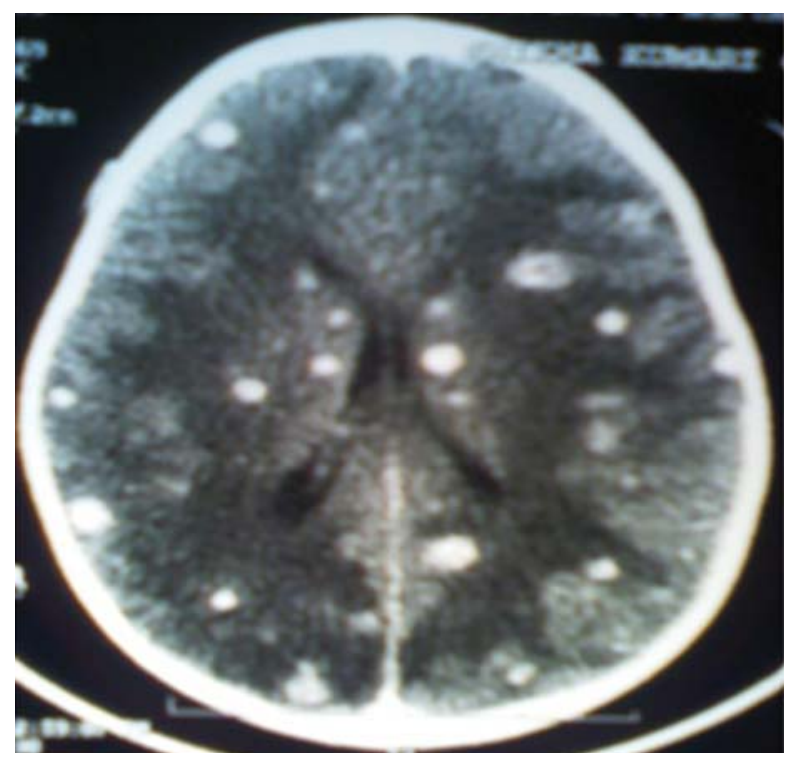

Fig 2: CT Brain after 4 weeks of Anti-tubercular treatment without steroid in a case of TBM revealed multiple neurotuberculoma with edema

\section{Discussion}

Neurological deterioration in patients with neuro-tuberculosis who are receiving ATT should alert clinicians to the possibility of paradoxical neurotuberculomas. A paradoxical response is defined as the clinical or radiological worsening of pre-existing tuberculous lesions or the development of new lesions not attributable to the normal course of disease, in a patient who initially improved with ATT. Tuberculous involvement of the central nervous system in our case (clinical meningitis) was evident at the initial presentation.

The paradoxical response to ATT, which usually develops after two weeks of treatment, is well known. Up to $10 \%$ patients with central nervous system TB report paradoxical response mostly if ATT not adjuncted with steroid during intensive phase, and this number may be as high as $30 \%$ in HIV-infected patients ${ }^{2}$. In our case, patient developed paradoxical result after 4 weeks of ATT, and most likely due to non prescription of steroid during intensive phase ATT. Patients recently started on ATT should be monitored for the development of paradoxical response. Most authorities recommend that these patients continue treatment with routine ATT.

The paradoxical response is a component of immune reconstitution inflammatory syndrome or immune restoration syndrome, which results from an exuberant inflammatory response toward incubating opportunistic pathogens ${ }^{3}$. T-cell suppression and anergy during active tuberculosis have been well recognized. This phenomenon is related in part to the production of interlukin-10 by peripheral blood T-lymphocytes during active tuberculosis. After completion of tuberculosis therapy, T-lymphocytes no longer produce interlukin-10. It is known that interlukin-10 down-regulates T-helper cell activity. Therefore, it is not surprising that successful treatment may reinstitute T-cell activity. It means that, over the course of tuberculosis treatment, a heightened immune response may occur despite the vanishing pool of viable mycobacteria ${ }^{4,5}$. This reversal of anergy state can explain the paradox of development of multiple neurotuberculomas despite institution of effective antituberculous drug therapy in our case.

Why does such phenomenon occur only in few patients with tuberculosis? This may be attributed to the differences in the immune responses of patients. There is sufficient evidence which showed that the host response to Mycobacterium tuberculosis plays a 
major role in determining the clinical manifestations and ultimate outcome of persons who encounter this pathogen ${ }^{6}$.

There are many reports suggesting resolution of paradoxical response with steroids ${ }^{7}$. In our case we added adjuvant steroid to ATT and continued ATT as per earlier regimen and patient symptomatically improved without any recurrence of seizure. The rationale behind the use of adjuvant steroids lies in reducing harmful effects of inflammation as the ATT kill the mycobacterium ${ }^{8}$.

\section{Conclusion}

This case emphasizes the importance of addition of steroid along with anti-tubercular drugs in the treatment of central nervous system tuberculosis. There is a need for paediatrician caring children at primary level to be aware of the occurrence of paradoxical reactions to ATT especially in CNS TB.

\section{References}

1. World health Organization. Global tuberculosis control. Report WHO. Geneva. 2011. Available: http://www.who.int/tb/publications/global_ report/2011/gtbr11_full.pdf. Accessed on January 22, 2014.
2. Gupta M, Bajaj BK, Khawaja G. Paradoxical response in patients with CNS tuberculosis. J Assoc Physicians India 2003;51:257-260.

3. Shelburne SA III, Hamill RJ. The immune reconstitution inflammatory syndrome. AIDS ReV 2003;5:67-79.

4. Bsussiotis VA, Tsai EY, Yunis EJ, et al. IL-10producing T-cells suppress immune responses in anergic tuberculosis patients. J Clin Invest 2000;105:1317-25.

5. Garcia M, Vargas JA, Castejon R. Flow-cytometric assessment of lymphocyte cytokine production in tuberculosis. Tuberculosis (Edinb). 2002;82:37-41.

6. Schluger NW, Rom WN. The host immune response to tuberculosis. Am J Respir Crit Care Med 1998;157:679 - 91.

7. Rodriguez-Bano J, Muniain MA, Aznar J, et al. Systemic paradoxical response to anti tuberculous drugs: resolution with corticosteroid therapy. Clin Infect Dis 1997;24:517-519.

8. Monga PK, Dhaliwal U. Paradoxical reaction in tubercular meningitis resulting in involvement of optical radiation. Indian J Ophthalmol 2009;57:139-41. 